\title{
Music Questions in Social Q\&A: An Analysis of Yahoo! Answers
}

Hertzum, Morten; Borlund, Pia

Published in:

Journal of Documentation

DOI:

10.1108/JD-02-2017-0024

Publication date:

2017

Document version

Peer reviewed version

Citation for published version (APA):

Hertzum, M., \& Borlund, P. (2017). Music Questions in Social Q\&A: An Analysis of Yahoo! Answers. Journal of Documentation, 73(5), 992-1009. https://doi.org/10.1108/JD-02-2017-0024 


\title{
Music Questions in Social Q\&A: An Analysis of Yahoo! Answers
}

\author{
Morten Hertzum \\ University of Copenhagen, Denmark, hertzum@hum.ku.dk
}

Pia Borlund

University of Copenhagen, Denmark, pia.borlund@hum.ku.dk

\begin{abstract}
Purpose - Social question and answer (social Q\&A) sites have become a popular tool for obtaining music information. This paper investigates what users ask about, what experience the questions convey, and how users specify their questions.
\end{abstract}

Design/methodology/approach - A total of 3897 music questions from the social Q\&A site Yahoo! Answers were categorized according to their question type, user experience, and question specification.

Findings - The music questions were diverse with (dis)approval (42\%), factual (21\%), and advice $(15 \%)$ questions as the most frequent types. Advice questions were the longest and roughly twice as long as (dis)approval and factual questions. The user experience associated with the questions was most often pragmatic (24\%) or senso-emotional (12\%). Pragmatic questions were typically about the user's own performance of music, while senso-emotional questions were about finding music for listening. Notably, half of the questions did not convey information about the user experience but the absence of such information did not reduce the number of answers. In specifying the questions, the most frequent information was about the music context and the user context.

Research limitations/implications - This study suggests a division of labor between social Q\&A sites and search engines for music information retrieval. It should be noted that the study is restricted to one social Q\&A site.

Originality/value - Social Q\&A sites provide an opportunity for studying what information real users seek about music and what information they specify to retrieve it, thereby elucidating the role of social Q\&A in music information seeking.

Keywords: music information retrieval, social Q\&A, information seeking, information behavior Article classification: research paper 


\section{Introduction}

Throughout the world people listen to, play, and otherwise take an interest in music to convey feelings, celebrate events, commemorate persons, create an atmosphere, state a protest, make a living, accompany other activities, and so forth. The multiple uses of music make music information seeking a diverse activity that is pursued using different tools. One of these tools is social question and answer (social Q\&A) sites. While research on music information retrieval has mainly addressed the automatic extraction of features from music files to make these features available for retrieval (Burgoyne et al., 2016, Schedl et al., 2014), social Q\&A sites provide an opportunity for studying what information real users seek and what information they specify to retrieve it. Such studies are necessary to understand the match between real information needs and the features that can be automatically extracted from music files and to investigate the role of social Q\&A in music information seeking. Several studies have stressed the need for more research on how real users seek and retrieve music information (e.g., Futrelle and Downie, 2003, Lee and Cunningham, 2013).

Social Q\&A (or community $Q \& A$ ) leverages the time and effort of everyday users to answer questions (Gazan, 2011). Once a question has been posted any user can answer it. In this study we target Yahoo! Answers, which is similar to other social Q\&A sites such as Answerbag and Quora but differs from ask-an-expert sites on which questions are answered by specialists, often for a fee. Due to the large amount of traffic on Yahoo! Answers, questions often receive multiple answers within a short period of time but also quickly disappear in the flood of new questions (Harper et al., 2008). The design of the site encourages users to browse questions by category, one of which concerns music. On the basis of manually categorizing 3897 music questions on Yahoo! Answers we analyze (a) the types of music questions users ask, (b) the user experience conveyed in the questions, and (c) the information supplied in specifying the questions. We supplement the categorization of the questions with information about their length and the number of answers. This information provides surrogate indicators of the amount of detail in the specification of the question and the amount of interest in the question from the user community, respectively.

Research on the seeking and retrieval of music information was sporadic until the late 1990s (Schedl et al., 2014), partly because the technologies underlying music information retrieval and social Q\&A are fairly recent. In the next section we review related work in music information retrieval and social Q\&A. We start this review by inserting music in an everyday-life context because the music questions we analyze predominantly arise from the users' everyday lives, as opposed to their professional careers. Then we describe the method we used in collecting and analyzing the data from Yahoo! Answers. To bolster the quality of the analysis we adopted an existing taxonomy (Harper et al., 2010) for the categorization of question types, slightly adapted a taxonomy of the customer experience (Gentile et al., 2007) to fit it to the user experience of music information retrieval, and devised our categorization of the information supplied in specifying the questions on the basis of existing empirical studies (e.g., Lee, 2010). In the remainder of the article we first present the results of the analysis and then discuss the results in relation to other work on music information retrieval and social Q\&A.

\section{Related work}

The following review covers research on how users seek and retrieve music information. Lee (2010, p. 1025) note that without a rich understanding of user needs and behaviors, research on music information retrieval runs the risk of developing "ill-suited systems for the users" and of remaining

"weak on evaluation and application to real users". Thus, user studies are important. 


\subsection{Uses of music in everyday life}

A consistent finding in studies of the uses of music in everyday life is that people consciously and actively use music as a resource and that their use of music differs across contexts (e.g., North et al., 2004, Sloboda et al., 2001). Music is rarely the main task in which people engage; rather they use music as accompaniment to other activities such as eating, exercising, homework, housework, and transport. DeNora (1999) found that people often used music to align themselves with the mental state appropriate for carrying out different tasks and exhibited considerable awareness of which music was effective for which tasks. Music has the capacity to change people's mood and they mostly exploit this capacity by listening to music that makes them more positive, aroused, and attentive (e.g., Sloboda et al., 2001). North et al. (2004) found that their respondents' reasons for listening to music were most often context-specific. For example, "it helped me to pass the time" was a frequent reason when respondents were on their own, at work, or with strangers. Conversely, "it helped to create the right atmosphere" was a frequent reason when they were with their boyfriend or girlfriend. Preferences for different music genres have not just been linked to different contexts but also to more stable factors such as personality (Rentfrow and Gosling, 2003) and social status (van Eijck, 2001). For example, Rentfrow and Gosling (2003) found that people who enjoyed energetic and rhythmic music (i.e., rap and hip-hop, soul/funk, and electronica/dance) tended to reject conservative ideals, be talkative, and consider themselves physically attractive whereas people who enjoyed reflective and complex music (i.e., classical, jazz, blues, and folk) tended to reject conservative ideals, be inventive, and consider themselves intelligent.

\subsection{Music information retrieval}

The starting point of most work on music information retrieval is that music information is increasingly available in digital form. By extracting musical features from digital music files it becomes possible to make the content of the music searchable. This way, users can search for music by specifying its rhythm, timbre, melody, loudness, or other features. Schedl et al. (2014) report that the algorithms for extracting such features have an accuracy of around $80 \%$, depending on the task. In addition to the music files, information about the context of the music is also increasingly available in digital form in terms of, for example, album artwork, chart ratings, sales statistics, and information about the artists' background. Such music-context information provides additional search options beyond those of content-based and conventional bibliographic searching (Knees and Schedl, 2013). A final type of information concerns the users and their context (Schedl et al., 2014). The users' demographics, mood, activities, social context, and spatio-temporal environment increasingly leave digital traces that may enable the personalized or context-aware retrieval of music information. In Figure 1 we summarize the four types of information considered in music information retrieval.

Insert Figure 1 about here

User studies in music information retrieval find that users engage in both goal-directed querying and open-ended browsing. The two types of search behavior are often interleaved (Cunningham et al., 2003), thus systems for retrieving music information should support querying and browsing in an integrated manner. A frequently recommended way of supporting browsing is by facilities for retrieving music that is similar to a specified piece of music (e.g., Braunhofer et al., 2013). The similarity may be in music content or in another of the information types in Figure 1. In addition to similarity, users often search for music information by specifying bibliographic information. For example, the top-five search options used by the respondents in the study by Lee et al. (2016) were performer $(95 \%)$, title $(90 \%)$, recommendations from other people $(77 \%)$, lyrics $(76 \%)$, and similar 
artist (70\%). While the use of bibliographic information such as performer and title was also prominent in earlier studies, the use of options for retrieving similar music has increased with the increasing availability of such options in systems for music information retrieval. In an earlier study, Bainbridge et al. (2003) found that bibliographic information (81\%) was, by far, the most frequently used search option, whereas users rarely described their needs by specifying similar music (5\%).

With respect to the desired outcome of the search process, Lee et al. (2016) asked respondents how likely they were to seek specified kinds of music information. The most frequently sought for music information was title (92\%), artist information (83\%), lyrics $(78 \%)$, and sample tracks for listening (75\%). These four kinds of music information were identical to the four top responses to the same question in an earlier survey by Lee and Downie (2004). When users were seeking music tracks for listening, Kamalzadeh et al. (2016) found that they wanted to be able to control the mood conveyed by the music and how familiar and distracting the music was. The users tended to prefer familiar music; during activities that required their attention they also wanted the music to be nondistracting. Laplante and Downie (2011) distinguished between utilitarian and hedonic outcomes and found that the utilitarian outcomes of music information seeking were to acquire music and information about music. Information about music was acquired to enrich the listening experience, increase music knowledge, and optimize future acquisitions. The hedonic outcomes consisted of taking pleasure in the information-seeking process itself and finding themselves deeply absorbed when seeking music. Several of the study participants considered searching for music on the web an engaging pastime and often lost their sense of time while doing it.

\subsection{Social Q\&A}

The hedonic qualities of engaging with others when looking for information about a topic of interest are pivotal to the success of social Q\&A, so much so that it is well-established that "some users seek information on SQA [social Q\&A] sites, while others seek conversation and contact" (Gazan, 2011, p. 2304). The affordance for seeking conversation and contact sets social Q\&A apart from ask-anexpert sites, such as Google Answers (discontinued in 2006), and conventional search engines. Several studies provide classifications of the questions asked in social Q\&A. In addition to the distinction between informational and conversational questions (e.g., Harper et al., 2009), studies frequently distinguish between fact-seeking, advice-seeking, and opinion-seeking questions (e.g., Adamic et al., 2008). In an effort to unify existing classifications Harper et al. (2010) proposed a classification with two future-oriented categories (advice and identification), two present-oriented categories (quality and (dis)approval), and two past-oriented categories (prescriptive and factual). They found that identification and (dis)approval questions were significantly less likely than the four other question types to produce answers of lasting value to others. We return to the classification by Harper et al. (2010) in the next section but also note that none of the question classifications mentioned above are specific to music. A typology specific to music, but unrelated to social Q\&A, has been offered by Rousi et al. (2016). They distinguish between five categories of music information: music listening, music making, graphic representations of music, technological models of music, and ideological models of music.

Choi and Shah (2016) investigated the users' motivations for asking questions on Yahoo! Answers and WikiAnswers. Users of Yahoo! Answers tended to seek opinion and advice, whereas users of WikiAnswers were more likely to ask fact-finding questions. Even when the users were aware of questions that might be similar to their own question and had already been answered they still wanted to ask their own question "to receive new personalized answers" (Choi and Shah, 2016, p. 1190). With specific reference to Yahoo! Answers, Shah (2011) found that the majority of the questions asked on this social Q\&A site got an answer within a few minutes but that it took longer to receive an answer that satisfied the asker. The criteria that determine satisfaction with answers on Yahoo! Answers have been studied by examining the reasons that users may provide when they, optionally, select one of the answers they have received as the best answer. This way Kim and Oh 
(2009) identified six classes of criteria: socio-emotional (30\%), content (26\%), utility (23\%), information source $(9 \%)$, cognitive (7\%), and extrinsic (4\%). Importantly, the criteria varied according to the topic of the question. For example, utility criteria were frequent for self-help questions and socio-emotional criteria dominated for music questions. Harper et al. (2008) found that paying \$10 (in October 2006) for an answer on Google Answers led to answers of better quality than asking a question on Yahoo! Answers. However, Yahoo! Answers produced answers at least as good as those returned from other social Q\&A sites, from Google Answers by paying $\$ 3$, and from library reference services.

In a study of music questions on Music StackExchange, Fu and Fan (2016) identified five question types: seeking guidance, seeking resources, seeking opinions, requesting research, and factual questions. Several of these question types, in particular seeking guidance, were about performing music rather than listening to it. Conversely, the research on music information retrieval and that on the seeking and use of music information in everyday life appear to presume a context of music listening. In a study of book requests on LibraryThing, Koolen et al. (2015) found that the most frequent kinds of information in the requests were information about the content of the book (74\%), examples of similar books (36\%), bibliographic information (28\%), and information about the reading experience evoked by the book (23\%). These requests were questions to other social Q\&A users, not search-engine queries. Specifying the content of a book, such as its topic or plot, resembles search-engine queries for books because book content is textual and, therefore, accessible to search engines to a much larger extent than music content is currently accessible to systems for music information retrieval. Conversely, providing examples of similar books resembles the use of recommender systems. Koolen et al. (2015) emphasized that the users often combined several kinds of information in their requests and that such combinations are not supported by current search/recommender systems. The possibility of freely combining different kinds of information in the questions may contribute to explaining the popularity of social Q\&A. Shah (2011) noted that among the 25 top-level categories on Yahoo! Answers the category Entertainment \& Music had the highest average number of answers per question, thereby suggesting that music information seeking is prominent in social Q\&A.

\section{Method}

The source of the data for this study was the Music category of Yahoo! Answers (answers.yahoo.com). Yahoo! Answers was launched in 2005 and with its millions of unique monthly users it is a widely used social Q\&A site (Gazan, 2011). We chose Yahoo! Answers for this study because it is widely used and because it is free of charge and, therefore, attracts a broad variety of users. It should however be noted that Yahoo! Answers is in English and thereby restricted to users with an English-speaking cultural background.

\subsection{Data collection}

The Music category of Yahoo! Answers is a subcategory of the top-level category Entertainment \& Music and itself further divided into ten subcategories: (1) Blues, (2) Classical, (3) Country, (4) Jazz, (5) Lyrics, (6) R\&B and soul, (7) Rap and hip-hop, (8) Rock and pop, (9) Singing, and (10) Other music. In January 2015, we downloaded the 500 most recent questions in each of the ten Music subcategories. The download was done using the tool import.io, which enabled us to specify which elements of the content of Yahoo! Answers pages we were interested in. Then, import.io grabbed these content elements and saved them in a spreadsheet. For each question, the downloaded data contained the question, the question date, the answer count, and the set of all answers. After removing 952 duplicate questions, the data comprised 4048 questions. 


\subsection{Data analysis}

We analyzed the questions by categorizing them according to three classifications, see Table 1 . The first classification addressed the rhetorical content of the questions by categorizing them into the six question types proposed by Harper et al. (2010): advice, identification, (dis)approval, quality, prescriptive, and factual. The six question types have been found to capture virtually all questions on social Q\&A sites (Harper et al., 2010). In addition, this classification subsumed earlier taxonomies, such as the distinction between conversational and informational questions (e.g., Kim, 2010 ) in that conversational roughly equates identification and (dis)approval questions whereas informational roughly equates the remaining four question types. The second classification elaborated the experiential dimension of the questions. Among the models of customer or user experience we chose the one by Gentile et al. (2007) because it is comprehensive and its six components are well described. However, we collapsed the sensorial and emotional components into one category, senso-emotional, because we considered it impossible to distinguish reliably between them on the basis of the music questions. The resulting user-experience classification had five categories: senso-emotional, cognitive, pragmatic, lifestyle, and relational. The third classification concerned the information provided in the question, that is the question specification. This classification captured the majority of the features identified by Lee (2010) but at a more aggregate level. We distinguished between the four categories previously depicted in Figure 1: bibliographic, music content, music context, and user context. The bibliographic category contained attributes normally searchable in music databases, and the music-content category contained most of the additional attributes targeted by current systems for music information retrieval. The musiccontext and, in particular, user-context categories contained attributes that it mainly made sense to specify because the questions were addressed to people.

The process of categorizing the questions involved six steps. First, the questions were divided into four sets: a training set of 700 questions, a test set of 300 questions for assessing the reliability of the categorization, and two sets of 1524 questions each. The training and test sets were created by randomly selecting 70 , respectively 30 , questions from each of the ten subcategories of music questions. Second, the 700 questions in the training set were categorized by both authors independently. For the question-type and user-experience classifications each question was assigned to the category that best matched the question or to an 'other' category if none of the categories matched the question. For the question-specification classification each question was assigned to those zero, one, or several categories that matched the question. Third, all disagreements in the authors' categorizations of the training set were discussed to reach consensus about the categorization of the questions and create a shared understanding of the classifications. Fourth, the 300 questions in the test set were categorized by both authors independently. Cohen's (1960) Kappa of the agreement between the authors in their coding of the test set was .68, .64, and .86 for the question-type, user-experience, and question-specification classifications, respectively. That is, all three Kappa values were above the threshold of .60 recommended by Lazar et al. (2010) as indicating satisfactory reliability. Inspection of the confusion matrices showed that $49 \%$ of the disagreements about the question type involved the (dis)approval category and that $80 \%$ of the disagreements about the user experience involved the 'other' category. Fifth, all disagreements in the authors' categorizations of the test set were discussed and a consensus was reached. This served to bolster the authors' shared understanding of the classifications further. Sixth, on the basis of the shared understanding of the classifications built during the preceding steps each author categorized the questions in one of the two remaining sets of 1524 questions.

Insert Table 1 about here 


\section{Results}

The categorizing identified 151 (3.7\%) questions that were not about music but, for example, about sexual issues, simple math problems (e.g., "What is $1+1 \times 0+1$ ?"), or nothing at all (e.g., "Hey there?"). These questions were removed, leaving 3897 questions for the subsequent analysis.

\subsection{Question type}

Table 2 shows the distribution of the 3897 questions on the question types. The questions were not evenly distributed on the categories, $\chi^{2}(6, N=3897)=3170, p<.001$.

Questions about (dis)approval were the most frequent, and they were predominantly about approval rather than disapproval. A typical question in this category would ask for other users' favorite music (e.g., "What are your favorite songs?") and sometimes offer the asker's favorites in return (e.g., "Mine are Michael Jackson, Bob Marley and Stevie Wonder"). When the motivation for these questions was stated, it was often that askers wanted to listen to a new music genre (e.g., "Ive just recently started getting into country music and I don't know where to find good songs"), were updating their playlists (e.g., "Just looking for new songs for my 'classy' playlist"), or needed music for a special occasion (e.g., "I've been thinking about my wedding lately, and I was wondering if anyone knew of any songs someone could sing while I walk down the isle").

Insert Table 2 about here

The second largest category was factual questions. A large number of these questions were about identifying a musician (e.g., "Who is the guitarist for Summertime on Cheap Thrills by Big Brother and the Holding Company?"), an instrument (e.g., "Could someone name the instrument in this song?"), or a song (e.g., "What is the sexy striptease song that sounds like pink panther?"). At times users were asked to identify songs on the basis of very partial cues (e.g., "WHAT S THE SONG THAT GOES Duuuuuuuuuuuuuuuuuuuuuuun dun dun dun dun dun dun dun dun dun dun dun dundun dun dundundun dun?"). There were also factual questions that appeared to be about the askers' own musical behavior, such as their singing (e.g., "Does braces affect your singing?") or music acquisition (e.g., "What are good free music downloading websites?").

Advice questions were the third largest category of question types. Typical questions in this category were about which instrument to play (e.g., "Should i learn the cello or violin?"), which guitar to buy (e.g., "What is a good acoustic guitar for a beginner with small hands?"), and how to learn to play it (e.g., "Im 14 and I really wanna learn to play the guitar preferably country music, I just have no idea where to start?"). Other advice questions related to uncertainty about how to behave and what to expect at various musical events (e.g., "Female going to black metal concert alone [...] Thing is i'm female and 5'1. Will I be sacrificed or something?"). Music also created parent-teenager conflicts. Advice on these conflicts was mostly sought by the teenagers (e.g., "How do I convince my parents to let me go to a concert?") but occasionally also by the parents (e.g., "My teen listens to innapropriate music what do i do?").

The fourth largest category was identification questions, many of which expressed opinions and appeared to be a search for others with similar opinions rather than for answers (e.g., "Anyone else think RnB is terrible?"). The opinions were often negative and sometimes supplemented with sub questions about how long the bad state would last (e.g., "Why is music so horrible nowadays? When will it get better?"). Questions driven by positive opinions were fewer but included some about creating longer-lasting relations, such as fan communities (e.g., "I started up a Avenged sevenfold fan club on facebook and I can't get any likes. Can you please like and share my page") or bands (e.g., "Anyone want to start a band? [...] I really want to start a girl band and go on $\mathrm{X}$ factor"). 
There were also questions that related to the community of Yahoo! Answers users, for example by explicitly polling their opinion (e.g., "Poll: For a rock song to be extraordinary, do not the lyrics have to be great poetry unto themselves when read out loud without music?").

The prescriptive questions resembled the advice questions with the important difference that prescriptive questions presumed that a solution already existed. Thus, prescriptive questions searched for preexisting solutions, whereas advice questions searched for answers based on which the asker could generate a solution. Most of the prescriptive questions were about the practice of playing music, such as learning to play an instrument (e.g., "What are the steps in learning to play the piano?"), setting up the gear (e.g., "how do you apply a neck pickup on your guitar?"), producing specific sound effects (e.g., "How do Guitarists get that long sustained notes out of the guitar?"), and playing from music sheets (e.g., "How to play the places where there are 2 notes on the same vertical spot in this sheet music?"). Some questions explicitly asked for steps or rules (e.g., "Are there any rules for knowing what scales can be played over a key?") but most were phrased in a how-to fashion. At times, prescriptive questions bordered on advice questions (e.g., "I have a solo tomorrow for a jazz concert in a rather large auditorium. I'm super nervous! Any tips?").

The least frequent question type was quality questions. They resembled (dis)approval questions except that quality questions presumed the answer would, in essence, be objectively grounded rather than subjective opinion. Most of the quality questions were about the topic of songs (e.g., "Songs about being hurt by someone you thought you could always trust?") or the language used in the lyrics (e.g., "I kinda want to do the talent show but I wanna do an Avril L song and I can't find one with no curse words can you guys help me"). Other questions concerned music with qualities that made it suited for specific activities (e.g., "Whats the best music to listen to when meditating?") or equated religious beliefs with objective grounding (e.g., "Is it a sin to listen to and/or play blues music?").

The six question types captured nearly all questions; only $1 \%$ of the questions fell into the 'other' category (e.g., "Symphony Orchestra outfit?").

The questions were an average of $41.30(S D=46.08)$ words long. However, question length varied by question type, $F(6,3890)=72.98, p<.001$, see Table 2 . The question type explained $10 \%$ of the variance in question length. Bonferroni-adjusted pairwise comparisons showed that advice, prescriptive, and identification questions were longer than the other question types and that advice questions were longer than all but prescriptive questions. Possibly, advice and prescriptive questions required more explanation of the context to enable the user community to provide relevant answers. Identification questions might have motivated more detail to engage the community in longer discussions. None of the other pairwise comparisons were significant.

The number of answers also varied by question type, $F(6,3890)=2.67, p=.014$. However, the effect size was negligible in that the question type explained only $0.4 \%$ of the variance in the number of answers. To the extent that the number of answers indicated how interesting or popular a question was, interest or popularity was largely unaffected by question type. Bonferroni-adjusted pairwise comparisons showed that (dis)approval questions received more answers than advice questions. The standard deviation of the number of answers to (dis)approval questions was several times larger than that of the other question types, indicating that a small number of (dis)approval questions received many answers. The maximum number of answers was 1034 for the (dis)approval question "What is a song that you keep playing over and over again?" For the other question types the maximum number of answers was 173 to the factual question "What is a song with 'Cigarette' or 'Cigarettes' in the title or lyrics?".

\subsection{User experience}

Table 3 shows the distribution of the questions on the user-experience categories. The questions were not evenly distributed on these categories, $\chi^{2}(5, N=3897)=4105, p<.001$. 
Insert Table 3 about here

The largest category in this classification was the 'other' category. As much as $52 \%$ of the questions did not convey a user experience to those who read and answered the questions. We are not implying that no experience was associated with asking these questions, merely that it was not conveyed in the question. In terms of question type the majority of the advice (89\%) and prescriptive (86\%) questions conveyed a user experience; for the other question types at least $51 \%$ of the questions did not, see Table 4.

Insert Table 4 about here

Pragmatic questions were the most frequent user experience. Many of these questions were about playing an instrument, such as whether to start on a new instrument (e.g., "Can i learn the trumpet at 23 ?"), how to practice (e.g., "How can I become a better singer?"), and what songs to play (e.g., "I need a song to perform on a Ukulele at my school talent show"). There were also questions about listening to music (e.g., "Is listening to music using earphones dangerous?"), using music to support other activities (e.g., "I need some music to pump me up before duels is wrestling"), and having music as a source of income (e.g. "Playing a gig at a coffee house. should a percentage of tips go to the house?"). The majority of the prescriptive (78\%) and advice (70\%) questions were pragmatic; for the other question types at most $18 \%$ were pragmatic (see Table 4 ).

The second most frequent user experience was senso-emotional. As much as $65 \%$ of the sensoemotional questions were of the (dis)approval type. Many askers looked for songs that matched their current mood or situation (e.g., "Songs about giving up in life or giving life another chance? Ive been really down lately and need some music to listen to that really fits my life"). Most of these questions asked for sad songs. Others were looking for songs to cheer them up (e.g., "Songs that help trying to be able to love again?"), help them relax (e.g., "Calming piano music for anixety/stress?"), create a romantic atmosphere (e.g., "Songs to make love too?"), or get a party going (e.g., "What are some blues songs that make people want to dance?"). These questions indicate that music was seen as contributing to positive senso-emotional changes.

In 222 questions the user experience was relational. Most of these questions were requests for songs to let a loved one know about the asker's feelings (e.g., "What's a good song to tell a girl you like her?") or to get over being left by a loved one (e.g., "Does anyone have any songs for when your crush is dating someone else?"). Some of these questions were about loving persons who were damaging themselves (e.g., "Songs about loving an addict?"). There were also relational questions about problems in parent-children relationships (e.g., "My parents don $\mathrm{t}$ support my dream to be a singer?"), about being honest in friendships (e.g., "How do i tell my friend she's a bad singer?"), and about forming a band to play together (e.g., "I want to start a band but dont know where to start?"). The questions with a relational user experience were predominantly (dis)approval (41\%) and advice (28\%) type questions.

Cognitive questions were almost as frequent as relational questions. As much as $50 \%$ of the cognitive questions were factual and another $24 \%$ were identification questions. The cognitive questions were mostly about the meaning of lyrics (e.g., "What does this song lyric mean?"), the name of artists (e.g., "What singer sang Candle in the Wind?"), and the term for musical items and operations (e.g., "What's that fancy piano thing you do where you piano a bunch of notes with both hands and you go from the top of the keys downwards? Is there a name for that?"). There were also 
questions about copyright (e.g., "Will Glenn Miller's music be public domain in 2014?"), the rules of composition (e.g., "Are there any rules for knowing what scales can be played over a key?"), and school assignments (e.g., "How many instruments are in a quartet? It's a school thing").

The least frequent user experience was lifestyle questions. For no question type did more than $5 \%$ of the questions express a lifestyle user experience (see Table 4). Most of the lifestyle questions were about reconciling the askers' musical preference with their background (e.g., "Is smooth jazz a 'white' genre? I love smooth jazz and I'm African American"), belief system (e.g., "What are some good Christian alternatives to these bands?"), or self-image (e.g., "Im Satanist but I like country is that a bad combo?"). Another group of lifestyle questions were about pursuing a career in music (e.g., "Do you think 24 is too old to pursue a professional music career?"). In addition, several questions concerned attaining stylish credibility (e.g., "I know some rappers purposely went to prison to boost they rap cred. Will it have the same effect for me, and if so, which crime should I commit so I'll only go away for 6 months max").

Question length varied with user experience, $F(5,3891)=172.33, p<.001$. The user experience explained $18 \%$ of the variance in question length. Bonferroni-adjusted pairwise comparisons showed that lifestyle and relational questions were longer than pragmatic and cognitive questions, which in turn were longer than senso-emotional questions. The 'other' questions were shorter than the questions in every user-experience category.

The number of answers also varied with user experience, $F(5,3891)=3.25, p=.006$. However, the user experience explained only $0.4 \%$ of the variance in the number of answers. To the extent that the number of answers indicated how interesting or popular a question was, interest or popularity was largely unaffected by the user experience expressed in the question.

\subsection{Question specification}

Table 5 shows the categories of information provided in specifying the questions. In contrast to the question-type and user-experience classifications, each question could be in zero, one, or several of the question-specification categories.

Insert Table 5 about here

Music context information was by far the most frequent question-specification category, mainly because users of Yahoo! Answers must ask their question in a sub category of the music category and most of these sub categories were a music genre, thereby making genre the most frequently specified piece of music context information. Music context information was present in $72 \%$ of the questions. There was a significant effect of question type on music context information, $F(6,3857)=$ $4.40, p<.001$. Bonferroni-adjusted pairwise comparisons showed that music context information was more often present for identification (84\%) questions than for advice $(62 \%)$ and factual $(67 \%)$ questions. We found no effect of user experience on music context information, $F(5,3857)=0.88, p$ $=.497$.

User context information was present in $46 \%$ of the questions and included various different kinds of information, for example about the asker's musical preferences, gear, experience, or aspirations. We found no effect of question type on user context information, $F(6,3857)=1.10, p=.359$, but there was a significant effect of user experience, $F(5,3857)=71.35, p<.001$. Bonferroni-adjusted pairwise comparisons showed that user context information was more often present for relational (95\%), lifestyle (88\%), and pragmatic (87\%) questions than for cognitive $(53 \%)$ questions and less often present for questions that did not convey a user experience $(17 \%)$ than for every userexperience category. 
Bibliographic information was present in $38 \%$ of the questions and mostly consisted of artist names, song titles, and album titles. There was a significant effect of question type on bibliographic information, $F(6,3857)=9.09, p<.001$. Bonferroni-adjusted pairwise comparisons showed that bibliographic information was more often present for identification (51\%) and (dis)approval (44\%) questions than for advice (19\%) and prescriptive (11\%) questions. We found no effect of user experience on bibliographic information, $F(5,3857)=1.33, p=.249$.

Music content information was present in $29 \%$ of the questions and, thereby, the least frequent question-specification category. It mostly specified an instrument or a snippet of the lyrics. There was a significant effect of question type on music content information, $F(6,3857)=9.76, p<.001$. Bonferroni-adjusted pairwise comparisons showed that music content information was more often present for advice (39\%) and factual (40\%) questions than for identification (17\%) and (dis)approval (18\%) questions. We also found a significant effect of user experience on music content information, $F(5,3857)=4.44, p<.001$. Bonferroni-adjusted pairwise comparisons showed that music content information was more often present for pragmatic $(45 \%)$ questions than for lifestyle $(10 \%)$ and 'other' (24\%) questions.

Question length increased significantly with the presence, as opposed to absence, of bibliographic, music content, music context as well as user context information, $F s(1,3895)=52.70,333.05$, 73.88 , and 1246.73 , respectively (all ps $<.001$ ). User context information explained $24 \%$ of the variance in question length and music content information explained $7.9 \%$. Bibliographic $(1.3 \%)$ and music context (1.9\%) information explained comparatively less of the variance in question length.

The number of answers decreased significantly with the presence, as opposed to absence, of music content information, $F(1,3895)=5.16, p=.023$, and user context information, $F(1,3895)=12.57, p$ $<.001$. However, the effect size was negligible in that music content information explained only $0.1 \%$ and user context information only $0.3 \%$ of the variance in the number of answers. There was no effect of bibliographic information, $F(1,3895)=0.16, p=.693$, and music context information, $F(1,3895)=0.46, p=.499$, on the number of answers.

\section{Discussion}

The analyzed questions predominantly arise from the users' everyday lives and the questions are asked to a community that is open to all information seekers. We note that music information seeking in this everyday-life context is a topic different from the information seeking of music students (e.g., Dougan, 2012), musicians (e.g., Kostagiolas et al., 2015), and music scholars (e.g., Brown, 2002).

\subsection{Music questions in social Q\&A}

The music questions were diverse with $42 \%$ (dis)approval, $21 \%$ factual, $15 \%$ advice, $13 \%$ identification, $6 \%$ prescriptive, and $4 \%$ quality questions. Using the same question types Harper et al. (2010) also found substantial diversity in their study of 300 questions randomly selected from three social Q\&A sites (Answerbag, Ask Metafilter, and Yahoo! Answers). However, (dis)approval questions were much more frequent for music, and identification questions were much rarer. While the (dis)approval questions typically concerned which songs people favored listening to, the advice and prescriptive questions often concerned the users' own performance of music. The performance of music is near absent in previous studies of the retrieval and use of music information in everyday life (e.g., North et al., 2004, Schedl et al., 2014, Cunningham et al., 2003). Instead these studies presume a context of music listening. Only $1 \%$ of the questions could not be assigned to any of the six question types, thereby corroborating the finding by Harper et al. (2010) that the six types capture virtually all questions on social Q\&A sites. 
In contrast, $52 \%$ of the questions did not contain information that conveyed the user experience associated with asking them. Thus, for every second question it remained unknown to the Yahoo! Answers community whether the information seeker asked the question for senso-emotional, cognitive, pragmatic, lifestyle, or relational reasons. When the questions did convey a user experience it was most often pragmatic $(24 \%)$ or senso-emotional $(12 \%)$. While the pragmatic questions typically concerned the information seeker's own performance of music, the sensoemotional questions were typically about finding music for listening. The users mostly wanted to listen to music that matched their current mood or could contribute to positive senso-emotional changes. Choi and Shah (2016) found that cognitive need was the most prominent motivational factor for asking a question on Yahoo! Answers and WikiAnswers. To the extent that user experiences can be likened with needs, cognitive questions were much rarer for music than for Yahoo! Answers in general. In addition, tension-free need (i.e., having fun, filling time, and emotional release) and affective need ranked second and fourth, respectively, of the five need categories analyzed by Choi and Shah (2016). That is, these two need categories were about as prominent for Yahoo! Answers in general as senso-emotional questions were for music. Surprisingly, none of Choi and Shah's five need categories capture pragmatic user experiences, the most common user experience in our analysis of music questions.

Contrary to earlier studies (e.g., Bainbridge et al., 2003, Lee, 2010), the music questions were not primarily specified by means of bibliographic information. A reason contributing to this finding is that music questions on social Q\&A are often about topics other than the retrieval of a specific song, artist, or concert performance. Bibliographic information is, for example, irrelevant to a question about how to apply a pickup on your guitar. Accordingly, fewer advice and prescriptive questions than (dis)approval questions contained bibliographic information. Across all question types, information about the music context and the user context was more frequent than bibliographic information, and information about the music content was less frequent. This suggests a division of labor between music questions on social Q\&A sites and search engines for music information retrieval. The search engines primarily aim to make the music content searchable in addition to the bibliographic information. For example, query by humming (Ghias et al., 1995) matches the query against the music content and appears to support music information retrieval in situations that are not well-suited for social Q\&A. Conversely, much information about the music context and user context is - currently - unavailable to search engines but often specified in the social Q\&A questions. The frequent presence of this information suggests that social Q\&A may be superior for the question types and user experiences that were most often specified by means of information about the music and user contexts, that is identification type questions and relational, lifestyle, and pragmatic user experiences.

Social Q\&A can be contrasted with ask-an-expert sites. Lee (2010) found that the majority of the music questions on the ask-an-expert site Google Answers were known-item searches. We found a broader mix of question types on Yahoo! Answers. While it may be unsurprising that the users more often ask their community at Yahoo! Answers than the experts at Google Answers about their favorite songs, it is noteworthy that the questions to the experts are mostly restricted to knownitem searches. Experts should also have the knowledge and experience necessary to offer competent advice, point to prescriptive solutions, and weigh the quality of an item. Such questions accounted for $25 \%$ of the questions in our analysis of Yahoo! Answers.

\subsection{Question length}

Consistent with Harper et al. (2010) advice questions were longer than the other question types, except prescriptive questions. Advice questions were roughly twice as long as (dis)approval and factual questions. We also found that lifestyle and relational questions were longer than questions conveying other user experiences, and that the presence or absence of information about the user context and music content contributed appreciably to explaining question length. The average 
length of the 3897 music questions was 41.30 words, which is shorter than the average length of questions in Harper et al. (2010), who analyzed randomly selected social Q\&A questions. Part of the explanation for the difference is that (dis)approval questions, which were among the shortest in both studies, were much more frequent for music than for social Q\&A in general. While longer questions may specify the information need in more detail, Morris et al. (2010) found that shorter questions received more useful answers and Shah et al. (2012) argue that long questions may overload potential respondents and discourage them from answering.

In a study of questions directed at film experts, Hertzum (2003) found an average question length of 111 words. This might suggest that questions posed to experts are longer than questions posed to a social Q\&A community. If asking the expert incurs a fee we suppose that the fee contributes to prolonging the questions because users want to make sure that the expert has all the information needed to provide a useful answer. Conversely, $54 \%$ of our music questions provided no information about the user context to assist potential respondents in tailoring their answer, and the absence of information about the user context shortened the questions. At the same time, submitting the music questions to a community of people makes for questions that are much longer than search-engine queries. For example, Zhang et al. (2009) found that queries for searching the Web contained an average of 2.9 terms. This length difference shows that people find it natural to write substantially more in seeking information from other people. It may also indicate that people have better intuitions about what another person will need to know in order to help them than about what kinds of information a search engine needs to perform effectively. Lastly, the music questions are presumably longer than search-engine queries because the users value the conversational element of social Q\&A.

\subsection{Number of answers}

The question type and user experience influenced the number of answers to the questions, and so did the presence or absence of information about the music content and user context. But the effect sizes were negligible. We consider it surprising that conveying a user experience was not associated with receiving more answers. Either the respondents inferred a user experience from implicit cues in the questions or their interest in answering a question was largely unaffected by the seeker's user experience. With respect to the question type our results discord with Harper et al. (2010), who found that the six question types explained $25 \%$ of the variance in the number of answers. In their study the explanatory power of the question type was mostly due to identification questions, which attracted more answers than quality, prescriptive, and factual questions. For music the proportion of identification questions was smaller and they did not, on average, attract more answers than the other question types. The (dis)approval questions attracted an average of 1.6 to 3.1 more answers than the other question types (see Table 2) but the standard deviation of the number of answers to (dis)approval questions was substantial and diminished their power in explaining the number of answers.

In their study of social Q\&A questions that attracted no answers Shah et al. (2012) report that the second most frequent attribute of these failed questions was lack of information. While we did not single out the questions that attracted no answers, the presence or absence of bibliographic information, music content information, music context information, and user context information did not appreciably affect the average number of answers to the music questions. Either the information seekers were competent at including the different kinds of information when it was relevant to their question or the respondents were prepared to answer the questions also when relevant information was missing. It appears likely that the large number of music questions that asked for advice and opinion, rather than facts, made it easier to provide an answer to incompletely specified questions. 


\subsection{Limitations}

Four limitations should be kept in mind when interpreting the results of this study. First, we analyzed the music questions of a single social Q\&A site. While Yahoo! Answers is a large social Q\&A site it would be valuable to repeat the study with different sites. These sites should, preferably, span users with cultural backgrounds and musical interests that differ from those of the users of Yahoo! Answers. Second, we lack the data to assess whether the 3897 analyzed questions are representative of the music questions on Yahoo! Answers. While the sample is large for a manual analysis, it is small compared to the full set of music questions on Yahoo! Answers. Our results should be applied with this proviso in mind. Third, we did not attempt a breakdown of our analysis on music genres. Preferences for different genres have been linked to personality (Rentfrow and Gosling, 2003) and social status (van Eijck, 2001), which might, in turn, lead to a different mix of music questions. While the sub categories of the music category provide a user-supplied genre indicator, we merely used the sub categories to sample a diverse set of questions for our analysis. We would hesitate to base a genre analysis on the sub categories and recommend that prior to such an analysis the sub categories be validated by an independent genre classification of the questions. Fourth, the answers to the questions were included in our analysis merely by relating the questions to the number of answers. We analyzed neither the content of the answers nor the temporal relationship between questions and answers. It appears likely that the question type, user experience, and question specification influence the content and timing of the answers. For example, relational questions might get more empathic answers and questions containing bibliographic information only might attract drier answers. Such analyses of the relationships between music questions and their answers are left for future work.

\section{Conclusion}

Social Q\&A sites such as Yahoo! Answers are popular means of obtaining information, including information about music. We have analyzed 3897 questions for music information with respect to their type, the user experience conveyed in the questions, and the information provided in specifying them. Four findings stand out:

- The most frequent music questions were (dis)approval, factual, and advice questions. Compared to studies of social Q\&A questions in general, (dis)approval questions, which typically asked the community of users about their favorite music, were more frequent for music and identification questions were rarer.

- The music questions sprang from contexts of performing as well as listening to music. The users' own performance of music dominated in advice and prescriptive questions and in questions conveying a pragmatic user experience. Conversely, (dis)approval and senso-emotional questions were mostly about finding music for listening.

- Half of the questions conveyed no user experience but attracted as many answers as the questions that did. The respondents' interest in answering a question may be as much about their own user experience in providing the answer as about the information seeker's user experience.

- More questions contained music context and user context than bibliographic information. This finding contrasts earlier studies and suggests that music questions in social Q\&A are more topically diverse than in other studied contexts, such as on ask-an-expert sites.

With respect to future work, there is a need for replicating our findings that many questions spring from a context of performing music and that only a minority of the questions contains bibliographic information because these findings are in contrast to previous work. In addition, we have not investigated whether music genre influences the question types and user experiences. Future work 
should also investigate how question types and user experiences influence the content and usefulness of the answers. Finally, we would welcome studies that compare social Q\&A with other platforms for music information retrieval, including music search engines. Such studies would refine our knowledge about the platform-dependence of question types, user experiences, and the information included in questions.

\section{Acknowledgements}

This study was conducted in the context of the CoSound project, which was co-funded by Innovation Fund Denmark (grant no. 0603-00475B). The funding source had no role in how the study was designed, conducted, reported, or published. Stavris Solo downloaded the music questions from Yahoo! Answers.

\section{References}

Adamic, L.A., Zhang, J., Bakshy, E. and Ackerman, M.S. (2008). "Knowledge sharing and Yahoo Answers: Everyone knows something", in WWW2008: Proceedings of the 17th International Conference on World Wide Web, ACM Press, New York, pp. 665-674.

Bainbridge, D., Cunningham, S.J. and Downie, J.S. (2003). "How people describe their music information needs: A grounded theory analysis of music queries", in Proceedings of the ISMIR2003 Conference of the International Society on Music Information Retrieval, ISMIR, pp. 221-222.

Braunhofer, M., Kaminskas, M. and Ricci, F. (2013), "Location-aware music recommendation", International Journal of Multimedia Information Retrieval, Vol. 2 No. 1, pp. 31-44.

Brown, C.D. (2002), "Straddling the humanities and social sciences: The research process of music scholars", Library \& Information Science Research, Vol. 24 No. 1, pp. 73-94.

Burgoyne, J.A., Fujinaga, I. and Downie, J.S. (2016). "Music information retrieval", in Schreibman, S., Siemens, R. and Unsworth, J. (eds.), A New Companion to Digital Humanities, Wiley, Chichester, UK, pp. 213-228.

Choi, E. and Shah, C. (2016), "User motivations for asking questions in online Q\&A services", Journal of the Association for Information Science and Technology, Vol. 67 No. 5, pp. 1182-1197.

Cohen, J. (1960), "A coefficient of agreement for nominal scales", Educational and Psychological Measurement, Vol. 20 No. 1, pp. 37-46.

Cunningham, S.J., Reeves, N. and Britland, M. (2003). "An ethnographic study of music information seeking: Implications for the design of a music library", in JCDL2003: Proceedings of the Third Joint Conference on Digital Llbraries, IEEE Press, Washington, DC, pp. 5-16.

DeNora, T. (1999), "Music as a technology of the self", Poetics, Vol. 27 No. 1, pp. 31-56.

Dougan, K. (2012), "Information seeking behaviors of music students", Reference Services Review, Vol. 40 No. 4, pp. 558-573.

$\mathrm{Fu}, \mathrm{H}$. and Fan, Y. (2016). "Music information seeking via social Q\&A: An analysis of questions in music StackExchange community", in JCDL2016: Proceedings of the Joint Conference on Digital Libraries, ACM Press, New York, pp. 139-142.

Futrelle, J. and Downie, J.S. (2003), "Interdisciplinary research issues in music information retrieval: ISMIR 2000-2002", Journal of New Music Research, Vol. 32 No. 2, pp. 121-131.

Gazan, R. (2011), "Social Q\&A", Journal of the American Society for Information Science and Technology, Vol. 62 No. 12, pp. 2301-2312.

Gentile, C., Spiller, N. and Noci, G. (2007), "How to sustain the customer experience: An overview of experience components that co-create value with the customer", European Management Journal, Vol. 25 No. 5, pp. 395-410. 
Ghias, A., Logan, J., Chamberlin, D. and Smith, B.C. (1995). "Query by humming: Musical information retrieval in an audio database", in MULTIMEDIA1995: Proceedings of the Third ACM International Conference on Multimedia, ACM Press, New York, pp. 231-236.

Harper, F.M., Moy, D. and Konstan, J.A. (2009). "Facts or friends? Distinguishing informational and conversational questions in social Q\&A sites", in Proceedings of the CHI2009 Conference on Human Factors in Computing Systems, ACM Press, New York, pp. 759-768.

Harper, F.M., Raban, D., Rafaeli, S. and Konstan, J.A. (2008). "Predictors of answer quality in online Q\&A sites", in Proceedings of the CHI2008 Conference on Human Factors in Computing Systems, ACM Press, New York, pp. 865-874.

Harper, F.M., Weinberg, J., Logie, J. and Konstan, J.A. (2010), "Question types in social Q\&A sites", First Monday, Vol. 15 No. 7.

Hertzum, M. (2003), "Requests for information from a film archive: A case study of multimedia retrieval", Journal of Documentation, Vol. 59 No. 2, pp. 168-186.

Kamalzadeh, M., Baur, D. and Möller, T. (2016), "Listen or interact? A large-scale survey on music listening and management behaviors", Journal of New Music Reserach, Vol. 45 No. 1, pp. $42-$ 67.

Kim, S. (2010), "Questioners' credibility judgments of answers in a social question and answer site", Information Research, Vol. 15 No. 1, paper 427.

Kim, S. and Oh, S. (2009), "Users' relevance criteria for evaluating answers in a social Q\&A site", Journal of the American Society for Information Science and Technology, Vol. 60 No. 4, pp. 716727.

Knees, P. and Schedl, M. (2013), "A survey of music similarity and recommendation from music context data", ACM Transactions on Multimedia Computing, Communications and Applications, Vol. 10 No. 1, pp. 2:01-2:21.

Koolen, M., Bogers, T., van den Bosch, A. and Kamps, J. (2015). "Looking for books in social media: An analysis of complex search requests", in ECIR2015: Proceedings of the European Conference on Information Retrieval, Springer, Berlin, pp. 184-196.

Kostagiolas, P.A., Lavranos, C., Korfiatis, N., Papadatos, J. and Papavlasopoulos, S. (2015), "Music, musicians and information seeking behaviour: A case study on a community concert band", Journal of Documentation, Vol. 71 No. 1, pp. 3-24.

Laplante, A. and Downie, J.S. (2011), "The utilitarian and hedonic outcomes of music informationseeking in everyday life", Library \& Information Science Research, Vol. 33 No. 3, pp. 202-210.

Lazar, J., Feng, J.H. and Hochheiser, H. (2010), Research methods in human-computer interaction, Wiley, Chichester, UK.

Lee, J.H. (2010), "Analysis of user needs and information features in natural language queries seeking music information", Journal of the American Society for Information Science and Technology, Vol. 61 No. 5, pp. 1025-1045.

Lee, J.H., Cho, H. and Kim, Y.-S. (2016), "Users' music information needs and behaviors: Design implications for music information retrieval systems", Journal of the Association for Information Science and Technology, Vol. 67 No. 6, pp. 1301-1330.

Lee, J.H. and Cunningham, S.J. (2013), "Toward an understanding of the history and impact of user studies in music information retrieval", Journal of Intelligent Information Systems, Vol. 41 No. 3, pp. 499-521.

Lee, J.H. and Downie, J.S. (2004). "Survey of music information needs, uses, and seeking behaviours: Preliminary findings", in Proceedings of the ISMIR2004 Conference of the International Society on Music Information Retrieval, ISMIR, Barcelona, pp. 441-446.

Morris, M.R., Teevan, J. and Panovich, K. (2010). "What do people ask their social networks, and why? A survey of status message Q\&A behavior", in Proceedings of the CHI2010 Conference on Human Factors in Computing Systems, ACM Press, New York, pp. 1739-1748.

North, A.C., Hargreaves, D.J. and Hargreaves, J.J. (2004), "Uses of music in everyday life", Music Perception, Vol. 22 No. 1, pp. 41-77. 
Rentfrow, P.J. and Gosling, S.D. (2003), "The do re mi's of everyday life: The structure and personality correlates of music preferences", Journal of Personality and Social Psychology, Vol. 84 No. 6, pp. $1236-1256$.

Rousi, A.M., Savolainen, R. and Vakkari, P. (2016), "A typology of music information for studies on information seeking", Journal of Documentation, Vol. 72 No. 2, pp. 265-276.

Schedl, M., Gómez, E. and Urbano, J. (2014), "Music information retrieval: Recent developments and applications", Foundations and Trends in Information Retrieval, Vol. 8 No. 2\&3, pp. 127261.

Shah, C. (2011), "Effectiveness and user satisfaction in Yahoo! Answers", First Monday, Vol. 16 No. 2.

Shah, C., Radford, M.L., Connaway, L.S., Choi, E. and Kitzie, V. (2012), "'How much change do you get from 40\$?" - Analyzing and addressing failed questions on social Q\&A", Proceedings of the Association for Information Science and Technology, Vol. 49 No. 1, pp. 1-10.

Sloboda, J.A., O'Neill, S.A. and Ivaldi, A. (2001), "Functions of music in everyday life: An exploratory study using the experience sampling method", Musicae Scientiae, Vol. 5 No. 1, pp. 9-32.

van Eijck, K. (2001), "Social differentiation in musical taste patterns", Social Forces, Vol. 79 No. 3, pp. 1163-1184.

Zhang, Y., Jansen, B.J. and Spink, A. (2009), "Time series analysis of a web search engine transaction log", Information Processing \& Management, Vol. 45 No. 2, pp. 230-245. 
Table 1. The three classifications used in categorizing the questions

\begin{tabular}{ll}
\hline Classification $\quad$ Category definitions \\
\hline Question
\end{tabular}

Advice

Identification

(Dis)Approval

Quality

Prescriptive

Factual

\section{User experience $^{b}$}

Senso-emotional

Cognitive

Pragmatic

Lifestyle

Relational

\section{Question specification}

Bibliographic

Music content

Music context
Directed at generating a new (or specifically tailored) solution, approach, or plan rather than locating or implementing an already existing solution. Grounded in the user's desire to inform future action

Directed at establishing a focused discussion (and potentially building relationships) among people with a shared commitment to a topic

Directed at encouraging readers to offer a 'favorite' or 'least favorite', with the implicit understanding that answers will be - at root - subjective opinions

Directed at seeking the 'best' or 'worst' example of a given class, or at weighing the relative merits of a given product, item, or concept, with the implicit understanding that answers will be - at root - objectively grounded

Directed at pursuing an already developed solution to a problem or challenge. Grounded in the user's desire to learn steps or strategies that are known (through experience) to address or resolve the issue at hand

Directed at seeking an answer that is objectively or empirically true, such as existing information, data, or settled knowledge

Involving the senses and the affective system. Music can arouse aesthetic pleasure, trigger a sense of beauty, generate moods, foster relaxation, energize and so forth

Involving thinking or conscious mental processes. Music may foster creativity, engage people in problem solving, or lead them to revise their ideas and assumptions

Involving the practical act of doing something. Music may be composed, rehearsed, performed, purchased, remixed, analyzed, discussed and so forth

Involving the affirmation of values and beliefs. Music may be associated with adhesion to certain values or with the affirmation of a social identity or lifestyle

Involving the user and his/her social context and relationships. Music may be consumed together with other people, be the core of a common passion, lead to the creation of a fan community and so forth

Information such as title, year, artist, composer, and publisher Information such as melody, lyrics, instruments, rhythm, key, and music video

Information such as genre, live performances, reviews, chart appearances, sales statistics, similar works, and artist background 

the answer is needed

Note. ${ }^{\text {a }}$ Adopted from Harper et al. (2010). ${ }^{\mathrm{b}}$ Adapted from Gentile et al. (2007) 
Table 2. Question types, $N=3897$ questions.

\begin{tabular}{lcccl}
\hline Category & Count & Percent $^{\mathrm{a}}$ & Length in words $^{\mathrm{b}}$ & Answers $^{\mathrm{b}}$ \\
\hline (Dis)Approval & 1628 & 42 & $32.11(35.59)$ & $7.96(27.28)$ \\
Factual & 799 & 21 & $30.15(33.28)$ & $5.96(7.18)$ \\
Advice & 573 & 15 & $68.05(57.59)$ & $5.14(1.70)$ \\
Identification & 498 & 13 & $54.74(59.66)$ & $6.39(3.80)$ \\
Prescriptive & 219 & 6 & $59.35(54.81)$ & $4.91(1.39)$ \\
Quality & 139 & 4 & $32.38(32.44)$ & $6.25(3.58)$ \\
Other & 41 & 1 & $20.32(25.17)$ & $5.88(2.99)$ \\
\hline
\end{tabular}

${ }^{a}$ Percentages sum to more than 100 due to rounding. ${ }^{b}$ Mean (standard deviation). 
Table 3. User experience, $N=3897$ questions.

\begin{tabular}{lrccl}
\hline Category & Count & Percent $^{\mathrm{a}}$ & Length in words $^{\mathrm{b}}$ & Answers $^{\mathrm{b}}$ \\
\hline Pragmatic & 920 & 24 & $62.64(48.88)$ & $5.09(1.98)$ \\
Senso-emotional & 462 & 12 & $46.28(46.60)$ & $6.18(2.96)$ \\
Relational & 222 & 6 & $74.50(61.43)$ & $5.70(2.38)$ \\
Cognitive & 218 & 6 & $57.63(59.04)$ & $5.61(2.64)$ \\
Lifestyle & 66 & 2 & $85.58(74.02)$ & $6.15(3.11)$ \\
Other & 2009 & 52 & $23.49(28.49)$ & $7.76(24.97)$ \\
\hline
\end{tabular}

a Percentages sum to more than 100 due to rounding. ${ }^{b}$ Mean (standard deviation). 
Table 4. Cross tabulation of question type and user experience, $N=3897$ questions.

\begin{tabular}{lcccccc}
\hline Question type & \multicolumn{7}{c}{ User experience } \\
\cline { 2 - 7 } & Pragmatic & Senso-emotional & Relational & Cognitive & Lifestyle & Other \\
\hline (Dis)Approval & 140 & 300 & 90 & 29 & 11 & 1058 \\
Factual & 141 & 29 & 20 & 109 & 7 & 493 \\
Advice & 400 & 14 & 63 & 8 & 26 & 62 \\
Identification & 51 & 90 & 31 & 53 & 17 & 256 \\
Prescriptive & 170 & 2 & 10 & 5 & 1 & 31 \\
Quality & 17 & 27 & 6 & 12 & 4 & 73 \\
Other & 1 & 0 & 2 & 2 & 0 & 36 \\
\hline
\end{tabular}


Table 5. Question specification, $N=3897$ questions.

\begin{tabular}{lcccc}
\hline Category $^{\mathrm{a}}$ & Count & Percent & Length in words $^{\mathrm{b}}$ & Answers $^{\mathrm{b}}$ \\
\hline Music context & 2818 & 72 & $45.19(49.92)$ & $6.56(8.16)$ \\
User context & 1800 & 46 & $65.79(50.84)$ & $5.57(2.90)$ \\
Bibliographic & 1467 & 38 & $48.15(51.46)$ & $6.53(4.45)$ \\
Music content & 1119 & 29 & $61.68(57.45)$ & $5.65(5.71)$ \\
\hline
\end{tabular}

${ }^{a}$ Each question can be in multiple categories. ${ }^{b}$ Mean (standard deviation). 


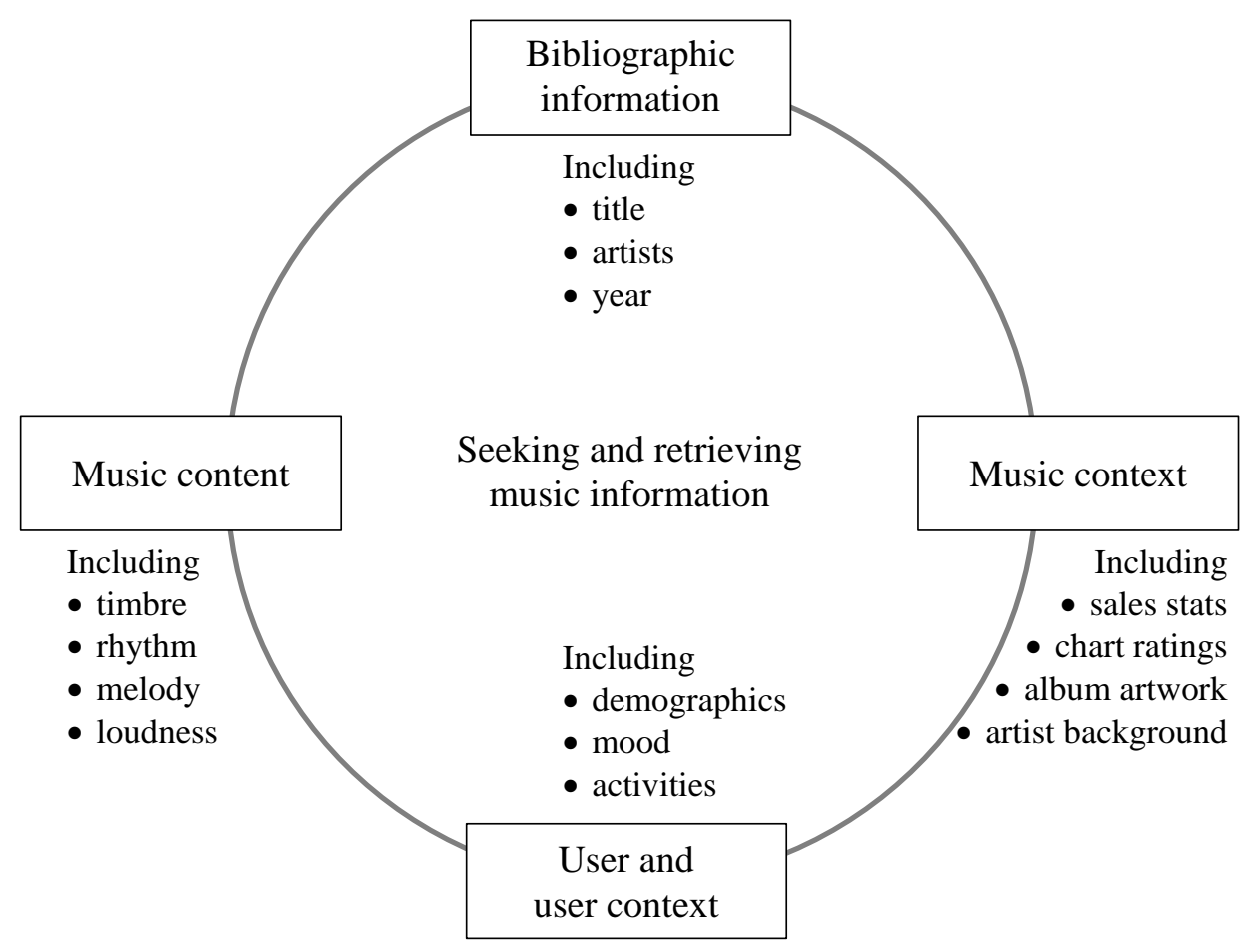

Figure 1. The four types of information considered in music information retrieval. 